\title{
Open adrenalectomy versus laparoscopic
} adrenalectomy for adrenocortical carcinoma: a retrospective comparative study on short-term oncologic prognosis

This article was published in the following Dove Press journal: OncoTargets and Therapy

\section{Guo-Yang Zheng \\ Han-Zhong Li \\ Jian-Hua Deng \\ Xue-Bin Zhang \\ Xing-Cheng Wu}

Department of Urology, Peking Union Medical College Hospital, Chinese Academy of Medical Sciences and Peking Union Medical College, Beijing, China
Correspondence: Han-Zhong Li Department of Urology, Peking Union Medical College Hospital, Chinese Academy of Medical Sciences and Peking Union Medical College, I Shuaifuyuan Road, Dongcheng District, Beijing 100730, China

Tel +86 I58 I095 94I5

Fax +86106915 2510

Email hzlipumch@163.com
Purpose: Open adrenalectomy (OA) remains the gold standard of surgical therapy for adrenocortical carcinoma, while the role of laparoscopic approach is controversial. We aim to explore the influence of surgical approaches on the oncologic prognosis of adrenocortical carcinoma by comparing the short-term outcomes of patients undergoing OA with those undergoing laparoscopic adrenalectomy (LA).

Patients and methods: We retrospectively analyzed the baseline characteristics, perioperative data and short-term prognosis of 42 patients diagnosed with stage I-III adrenocortical carcinoma, receiving OA $(n=22)$ and LA $(n=20)$ as primary therapy. The primary end point was the first recurrence.

Results: OA group had larger mean maximum diameter of tumor $(10.1 \pm 3.6$ versus $6.3 \pm 2.2 \mathrm{~cm})$ and lesser benefits in operative time, bleeding loss and postoperative hospital stay than laparoscopic group. Mean disease-free survival (DFS) of OA was 44.8 \pm 35.1 months, which was longer than $17.5 \pm 10.4$ months of LA, and the rate of 2-year DFS after primary surgery in the open group was higher than in the laparoscopic group (61.1\% versus $21.4 \%$, respectively). Rates of 1- and 3-year DFS showed no significant difference. All patients undergoing LA (11/11) showed local recurrent lesions at the first time of recurrence, while 5 of 13 patients undergoing OA did not show local recurrence $(P=0.03)$.

Conclusion: $\mathrm{OA}$ for adrenocortical carcinoma is superior to laparoscopic approach in terms of DFS and rate of 2-year DFS, in spite of the larger maximum diameter of tumors and lesser benefit during perioperation. After LA, patients are more likely to show local recurrent lesions at the first time of relapse.

Keywords: adrenocortical carcinoma, adrenalectomy, open surgery, laparoscopy, prognosis

\section{Plain language summary}

Adrenocortical carcinoma (ACC) is a rare malignant endocrine tumor. Patients diagnosed with ACC usually have poor oncologic outcomes, and those with advanced stage tumors usually die in 3 years. Surgical resection is the most important therapy and the first choice for primary ACC, if it is possible to be resected completely. Also, complete resection may predict better prognosis than incomplete resection. There are mainly two surgical approaches for ACC, which are open adrenalectomy (OA) and laparoscopic adrenalectomy (LA). Whether the surgical approach could affect prognosis of patients or not is still controversial. The medical team of Dr Han-Zhong Li and Dr Guo-Yang Zheng conducted a retrospective analysis aiming to explore the association between surgical approaches and short-term prognosis of ACC. They found that OA for ACC is superior to laparoscopic approach in short-term oncologic prognosis. Patients undergoing OA showed longer disease-free survival 
and lower recurrence rate than those receiving LA, although OA meant larger maximum diameter of tumors and lesser benefit during perioperation. Also, they found that after LA, patients are more likely to show local recurrent lesions at the first time of relapse, which was a novel finding. In summary, they recommend OA as the gold standard of surgical treatment for ACC. Laparoscopic approach could be tried for appropriate cases on the premise of complete resection.

\section{Introduction}

Adrenocortical carcinoma (ACC) is a rare malignant tumor, the incidence of which is $\sim 0.72-2$ per million per year, and is responsible for $0.2 \%$ of cancer-related deaths in the USA. ${ }^{1}$ The prognosis of ACC is poor, with a high recurrence rate associated with tumor stage. Patients with stage I-II could survive as long as 159 months, and those with stage II usually survive $>5$ years. ${ }^{2}$ However, the median survival of patients with stage III is $<5$ years, and those with stage IV are unlikely to survive $>1$ year. $^{3}$ The 5 -year survival of patients with stage I-II may be $>96 \%$, while that of patients with stage III and stage IV is $40 \%$ and $28 \%$, respectively. Another important influencing factor affecting the survival of patients with ACC is the condition of surgical resection. For patients with all stages, the recurrence rate of $\mathrm{R} 0$ resection is about $23 \%$, while that of $\mathrm{R} 1$ or $\mathrm{R} 2$ resection is up to $51 \%$ or even higher. ${ }^{6}$ Therefore, surgery is crucial for the treatment and prognosis of ACC, and complete resection is necessary, which could contribute to extending the diseasefree survival (DFS).

Open surgery remains the gold standard of surgical treatment of ACC, while the role of laparoscopic surgery for ACC is still under debate. Some retrospective series reported laparoscopic surgery might lead to higher rate of recurrence, peritoneal carcinomatosis and positive margin, compared with open approach. ${ }^{7,8}$ On the contrary, other researches indicated that there was no difference between open and laparoscopic approaches in cancer-specific survival, recurrence-free survival, frequency of tumor capsule violation and peritoneal carcinomatosis for localized ACC. ${ }^{9}$ Unfortunately, there have been no prospective comparative series reported so far, and current studies and cases included in researches are limited.

Therefore, new data are still needed to compare the oncologic prognosis of ACC between open and laparoscopic operations. In our study, we aim to explore the association between the prognosis of patients diagnosed with ACC and the approach of primary surgery (open surgery versus laparoscopy) by analyzing the clinical information of patients with ACC retrospectively.

\section{Patients and methods}

\section{Patients}

In this single-institution retrospective study, we reviewed the clinical information of 42 adult patients diagnosed with ACC admitted to Peking Union Medical College Hospital (PUMCH) from April 2003 to July 2015. Patients younger than 18 years or accompanied with other malignant tumors were excluded. All tumors were stage I-III according to the classification of the European Network for the Study of Adrenal Tumors. All patients accepted adequate imageological examinations to exclude patients with distant metastasis before surgery. All patients underwent complete open adrenalectomy (OA) or laparoscopic adrenalectomy (LA) as the first treatment of primary tumors by the same medical team in PUMCH, and all patients did not receive any other adjuvant therapy before primary operations and the first recurrence. OA and LA were performed in the standard procedure, and the resection scope covered complete tumor, whole adrenal gland and surrounding adipose tissue as much as possible. Multiple organ resection or lymph node dissection was performed in selected cases as the occasion required, and operations with $\mathrm{R} 1 / \mathrm{R} 2$ resection were excluded. Patients had re-examination 1-3 months after surgery for the first time, and then took regular follow-up examinations once or twice a year. This retrospective study has been approved by the PUMCH Institutional Review Board. All procedures performed in our retrospective studies involving human participants were in accordance with the ethical standards of the institutional and national research committee and with the 1964 Helsinki declaration and its later amendments or comparable ethical standards. All patients involved in this study have signed to approve the publication of their medical information, and provided written informed consent for the use of their medical records and data in research, which were saved in the medical records in our hospital.

\section{Design}

All 42 patients meeting the study entry criteria were divided into OA group and LA group, according to the surgical approach. We compared the baseline characteristics, perioperative data and short-term prognosis between the two groups. The baseline characteristics included age, gender, endocrine function of tumor, the maximum diameter of tumor, tumor stage and Ki-67 index by pathologic result. Perioperative data included operative time, bleeding volume, postoperative hospital stay and postoperative complications. 
Evaluation of short-term prognosis consisted of DFS, rate of 1-year DFS, rate of 2-year DFS and rate of 3-year DFS. Also, we compared all the recurrence sites between the two groups to analyze the characteristic of the first time of recurrence. The primary end point was the first recurrence after surgical treatment, and DFS was defined as time from the date of tumor resection to the first evidence of tumor progression, or the last follow-up without tumor lesions. The determination criteria of progression of ACC depended on recurrence of symptoms or signs, and local recurrence or distance metastasis by the imageological examination or endocrine examination. The survival condition after first recurrence was not focused in our study because the adjuvant therapies after first recurrence are various and difficult to be standardized, such as reoperation, mitotane, radiotherapy and so on.

\section{Statistical analysis}

All analyses were performed by using of Statistical Product and Service Solutions version 24.0 (IBM Corporation, Armonk, NY, USA) software. Results were described using mean $( \pm \mathrm{SD})$, median (range) or rate. Results of enumeration data were compared using chi-squared test or Fisher's exact test. Results of measurement data according to normal distribution and homogeneity of variance were compared using Student's $t$-test, while the others were compared by nonparametric test. DFS analysis was conducted using the Kaplan-Meier method, and differences between groups were assessed with log-rank statistics. Cox regressive model was used for multivariate analysis to adjust for surgical approach, tumor stage and maximum diameter of tumor. Statistical significance in this study was considered at $P \leq 0.05$.

\section{Results}

We have summarized the basic clinical features of 42 patients diagnosed with ACC, 22 cases in OA group (10 males and 12 females) and 20 cases in LA group (9 males and 11 females), in Table 1. The mean age of patients in OA group was $48.5 \pm 14.1$ years and that of LA group was $45.2 \pm 10.9$ years. The stage of tumors in the two groups showed no significant difference, neither did the endocrine function nor Ki-67 index of tumors. However, patients undergoing OA had larger maximum diameter of tumor than those undergoing LA $(10.1 \pm 3.6$ versus $6.3 \pm 2.2 \mathrm{~cm})$.

The perioperative data indicated patients might benefit more from LA (Table 2). Both the median operative time and the median postoperative hospitalization stay of OA group were longer than those of LA group. In addition, the median intraoperative blood loss of OA group was 800 (200-1,250) $\mathrm{mL}$, which was much more than that of LA group (70 [25-400] mL). There was no significant difference in postoperative complications between the two groups, but one patient undergoing OA died of multiple organ failure caused by hemorrhagic shock 3 days after surgery, in whom was found a $16 \mathrm{~cm}$ sized tumor of the right adrenal gland with inferior vena cava tumor thrombosis.

Patients undergoing $\mathrm{OA}$ benefited more than those undergoing LA in the short-term oncologic prognosis (Table 3 ). The number of patients available for evaluating postoperative prognosis was less than the initial number of cases included in the study because of the loss to follow-up and non-arrival at the time of evaluation. There were 35 patients available to evaluate the rate of 1 -year DFS, with $78.9 \%(15 / 19)$ in OA group and $62.5 \%(10 / 16)$ in LA group $(P=0.400)$. The

Table I Baseline characteristics of 42 patients with ACC treated with OA and LA

\begin{tabular}{|c|c|c|c|}
\hline Baseline characteristics & OA group & LA group & $P$-value \\
\hline No of patients & 22 & 20 & \\
\hline Age, years, mean \pm SD $(95 \% \mathrm{Cl})$ & $48.5 \pm$ I $4.1(42.2-54.7)$ & $45.2 \pm 10.9(40.1-50.3)$ & 0.400 \\
\hline Sex, n (male/female) & $10 / 12$ & $9 / 11$ & 0.900 \\
\hline Endocrine function, $\mathrm{n}(\%)$ & & & 0.200 \\
\hline Non-function & $9(40.9)$ & $9(45)$ & \\
\hline Cortisol & $6(27.3)$ & $8(40)$ & \\
\hline Sexual steroid & $4(18.2)$ & $0(0)$ & \\
\hline Cortisol plus sexual steroid & $3(13.6)$ & $3(15)$ & \\
\hline Max diameter, $\mathrm{cm}$ & & & 0.001 \\
\hline Mean \pm SD $(95 \% \mathrm{Cl})$ & $10.1 \pm 3.6(8.4-\mid I .6)$ & $6.3 \pm 2.2(5.3-7.6)$ & \\
\hline Tumor stage, $\mathrm{n}(\%)$ & & & 0.100 \\
\hline Stage I & I (4.5) & $5(25)$ & \\
\hline Stage II & $12(54.5)$ & II (55) & \\
\hline Stage III & $9(40.9)$ & $4(20)$ & \\
\hline Ki-67 index (\%), median (range) & $20(6.25-40)$ & $15(2.75-25)$ & 0.400 \\
\hline
\end{tabular}

Abbreviations: ACC, adrenocortical carcinoma; LA, laparoscopic adrenalectomy; OA, open adrenalectomy. 
Table 2 Perioperative data of 42 patients with ACC treated with OA and LA

\begin{tabular}{|c|c|c|c|}
\hline Perioperative indexes & OA group & LA group & $P$-value \\
\hline Operative time, minutes & & & 0.004 \\
\hline Median (range) & $175.0(\mid 44.0-198.5)$ & $130.0(\mid 00.0-153.5)$ & \\
\hline Bleeding volume, $\mathrm{mL}$ & & & 0.001 \\
\hline Median (range) & $800(200-1,250)$ & $70(25-400)$ & \\
\hline Postoperative hospitalization, days & & & 0.018 \\
\hline Median (range) & $9.5(7.0-12.5)$ & $7.0(6.0-8.0)$ & \\
\hline Complications, n (\%) & & & 0.800 \\
\hline Grade I-2 & $9(40.9)$ & $7(35)$ & \\
\hline Grade 3-4 & $0(0)$ & $0(0)$ & \\
\hline Death & I (4.5) & $0(0)$ & \\
\hline
\end{tabular}

Abbreviations: ACC, adrenocortical carcinoma; LA, laparoscopic adrenalectomy; OA, open adrenalectomy.

total number of assessable patients for 2-year DFS was 32, and the rate of 2-year DFS of OA group was $61.1 \%(11 / 18)$, which was better than that of LA group $(21.4 \%[3 / 14]$; $P<0.05)$. We could collect the exact data of 31 patients for 3-year DFS, which showed 23.5\% (4/17) of patients in OA group and $21.4 \%(3 / 14)$ of patients in LA group had no statistical difference between them. Of all the patients with tumor recurrence appearing for the first time after primary surgery, the OA group showed longer mean DFS of $44.8 \pm 35.1(26.1-63.5)$ months than that of LA group (17.5 \pm 10.4 [11.4-23.5] months), with a significant statistical difference (Figure 1). Also, of all the cases included in our study, OA still demonstrated longer DFS after primary surgery than LA (Figure 2). After adjustment for tumor stage and maximum diameter of the tumor, the OA group was still superior to LA group in DFS. Surgical approach was an independent risk factor for recurrence, which showed the relative risk for recurrence of LA group was 2.1 compared with OA group.

A new finding was made about the characteristic of the first recurrence after primary adrenalectomy. Of the 13 patients who showed recurrence after OA, 5 patients had local tumor bed recurrence, 5 patients had distant recurrence and 3 patients had both local and distant recurrence. All the 11 patients who showed recurrence after LA had local tumor bed recurrence, and 3 of the 11 patients had distant recurrence at the same time. We assume that patients with ACC undergoing LA are more likely to show local tumor bed recurrence as the first relapse, compared with OA patients $(P=0.03)$.

\section{Discussion}

\section{Surgical approaches affect short-term prognosis}

A new finding of our study is the characteristic of the first recurrence after surgery for ACC, which showed patients receiving LA were more likely to show local recurrent lesions surrounding the primary tumor bed at the first time of recurrence after primary surgery. In addition, we supplement detailed data of short-term postoperative prognosis of ACC, which contributes to exploring the effect of surgical approach on the prognosis of ACC. It has an important significance because the sample size of current researches is small due to the rarity of ACC. Our study supports the mainstream opinion that patients with ACC benefit more from open surgical approach in overall survival, recurrence-free survival, recurrence rate and positive margins, compared with LA. ${ }^{10}$ Our study showed that, although open approach might lead

Table 3 Short-term oncologic prognosis of patients undergoing OA and LA

\begin{tabular}{|c|c|c|c|}
\hline Short-term prognosis & OA group & LA group & $P$-value \\
\hline \multicolumn{4}{|c|}{ Disease-free survival, months } \\
\hline Mean \pm SD $(95 \% \mathrm{Cl})$ & $44.8 \pm 35.1(26.1-63.5)$ & $17.5 \pm 10.4(11.4-23.5)$ & 0.023 \\
\hline I-year DFS, n/T (rate) & $15 / 19(78.9 \%)$ & $10 / 16(62.5 \%)$ & 0.400 \\
\hline 2-year DFS, n/T (rate) & $11 / 18(61.1 \%)$ & $3 / 14(21.4 \%)$ & 0.036 \\
\hline 3-year DFS, n/T (rate) & $4 / 17$ (23.5\%) & $3 / 14(21.4 \%)$ & 0.600 \\
\hline First recurrence sites & 13 & 11 & 0.080 \\
\hline Local & 5 & 8 & \\
\hline Distant & 5 & 0 & 0.030 \\
\hline Local and distant & 3 & 3 & \\
\hline
\end{tabular}

Abbreviations: DFS, disease-free survival; LA, laparoscopic adrenalectomy; OA, open adrenalectomy; T, total cases available to be evaluated. 


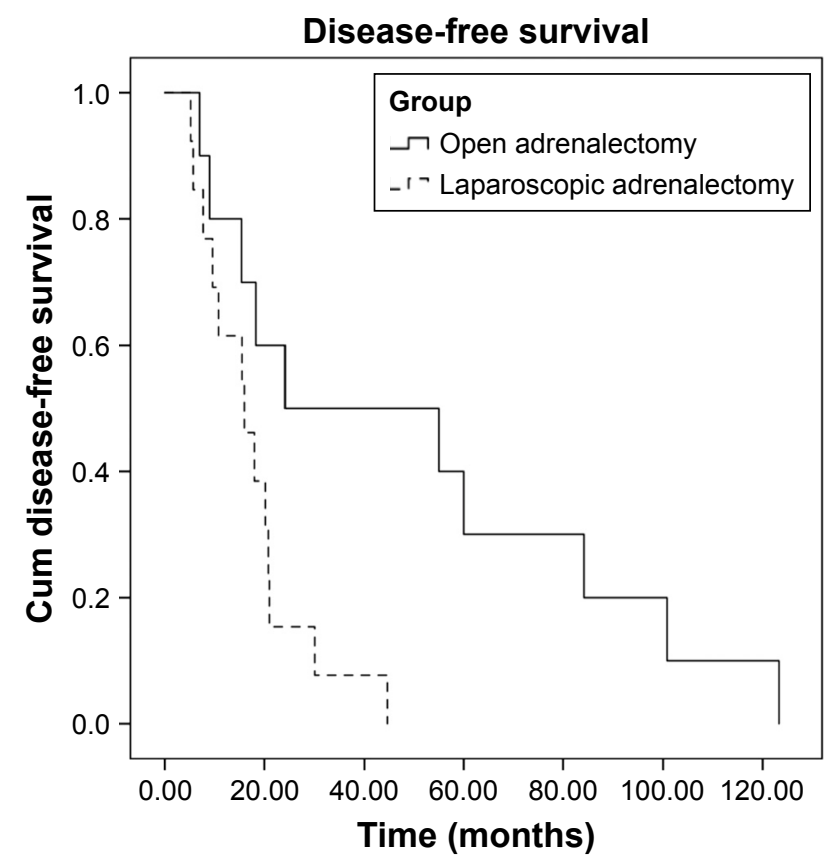

Figure I Kaplan-Meier analysis of disease-free survival of patients showing first recurrence after open adrenalectomy versus laparoscopic adrenalectomy.

to longer duration of operation, more intraoperative blood loss and longer postoperative hospitalization, the OA, indeed, could extend DFS and reduce the recurrence rate. This is important for the patients with ACC because they might suffer a lot from the symptoms and signs caused by the tumor, reducing their quality of life. What is worse, once the ACC recurs, the options and therapeutic effect of

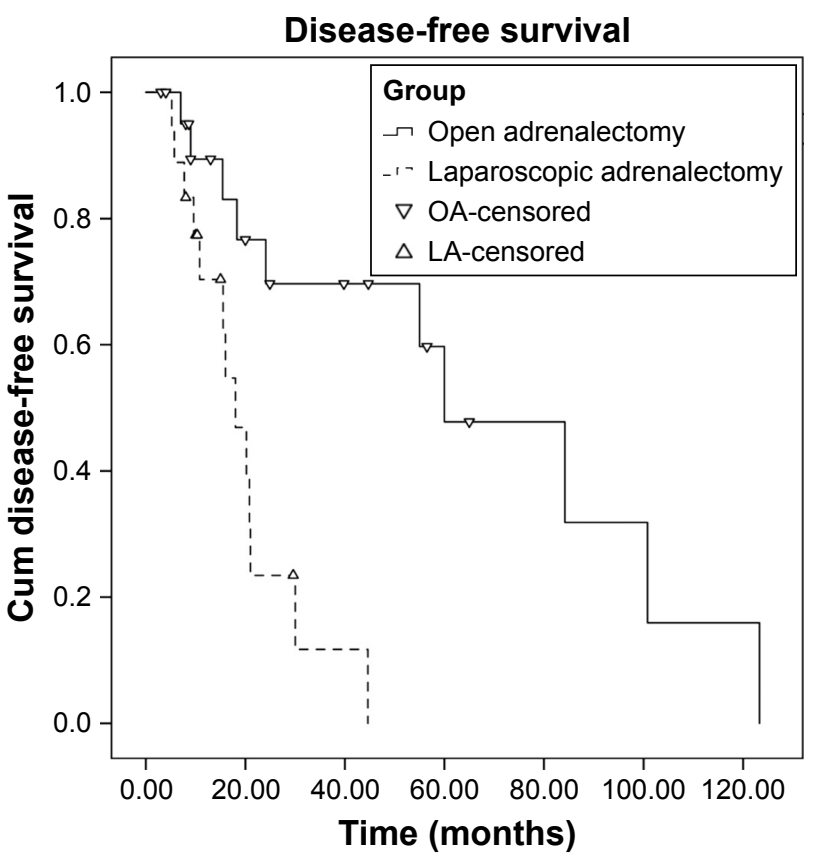

Figure 2 Kaplan-Meier analysis of disease-free survival of all the patients involved in the study undergoing open adrenalectomy $(\mathrm{OA})$ versus laparoscopic adrenalectomy (LA). sequential treatments are limited, which means acceleration of tumor progression and high cost of treatment. So, it is vital to prolong DFS after primary surgery for the management of ACC patients, and we recommend open surgical approach as the standard primary surgical treatment.

\section{$O A$ versus $L A$}

OA remains the gold standard of surgical treatment for ACC, which is superior to LA in the prognosis of patients with ACC, including overall survival, DFS, recurrence rate, rate of marginpositive and rate of peritoneal metastasis. ${ }^{7,8,11-13}$ A retrospective research published in 2013 included 256 patients undergoing $\mathrm{OA}$ for ACC and 46 cases receiving LA for ACC, which is the biggest number of cases reported currently. ${ }^{11}$ In this study, tumors resected by OA were significantly larger than those resected by laparoscopic approach, while patients undergoing $\mathrm{OA}$ had lower rate of positive margins than those receiving LA. Furthermore, after pathologic T stage was adjusted, the overall survival, recurrence-free survival and peritoneal recurrence-free survival of patients with $\mathrm{OA}$ were significantly longer than those receiving laparoscopic resection. Results of our study not only support the abovementioned conclusion but also make a detailed supplement on the short-term oncologic outcomes of 1-, 2- and 3-year DFS. In addition, a meta-analysis published in 2015 compared the overall survival between OA and LA. ${ }^{14}$ In that study, there were no statistically significant differences between OA and LA groups, considering the 2-, 3- and 4-year overall survival, while the 5-year overall survival was in favor of OA group.

On the contrary, several researches demonstrated that LA by an experienced surgeon was not inferior to OA with regard to oncologic outcomes, for selected cases of ACC without adjacent organ invasion or metastasis. ${ }^{9,15-17}$ A representative study published in 2010 included 152 patients diagnosed with ACC, and indicated that LA and OA did not differ with regard to the clinical prognosis of localized ACC with a diameter $<10 \mathrm{~cm} .{ }^{9}$ As the research presented, both diseasespecific survival and DFS showed no significant difference between patients undergoing LA and OA, and the clinical outcomes of patients converted to open surgery were not different from those in whom LA was completed. In addition, the overall survival and DFS of patients in LA group were not different from their respective matched OA controls. Similarly, rates of $\mathrm{R} 0$ resections, rupture of tumor capsule and peritoneal carcinomatosis were comparable between LA and OA.

Besides, it is commonly considered that LA has more advantages than $\mathrm{OA}$ in the perioperative benefits, such as 
operative time, blood loss, length of hospitalization, comfort degree and so on. Even some researches indicated that it might not be necessary to leave routine drainage after uncomplicated LA. ${ }^{18}$ All the patients included in our study received retroperitoneoscopic adrenalectomies, while laparoscopic transperitoneal adrenalectomy remains the safe and standardized procedure for adrenalectomy, with a short learning curve and low morbidity rate. As was demonstrated in a systematic review published by Conzo et al, ${ }^{19}$ retroperitoneal adrenalectomy may be comparable to laparoscopic transperitoneal adrenalectomy in operation time, pain score, blood loss, hospitalization, complications rates and return to normal activity. However, the transperitoneal approach may be superior to retroperitoneoscopic approach for adrenal tumors with size $>6 \mathrm{~cm}$. Another study demonstrated that LA of tumors with size $\geq 6 \mathrm{~cm}$ is more difficult, and the tumor size was positively correlated to the duration of surgery and intraoperative blood loss. ${ }^{20}$ The authors considered that LA was the gold standard of treatment for benign adrenal lesions and was safe and beneficial for patients. However, the results of LA depend not only on the experience of the single surgeon but also on the whole team involved in perioperative care. ${ }^{21}$ Therefore, this should also be considered when choosing the surgical approach.

On the basis of the identical clinical outcomes of these studies, some researchers insist that laparoscopic approach is feasible for localized ACC, but complete resection of tumor must be guaranteed. Most experts recommend LA as the appropriate treatment for ACC with diameter $<6$ $\mathrm{cm}$. Especially for the benign adrenal tumors such as adenomas causing Cushing syndrome or subclinical Cushing syndrome, LA has been confirmed as a safe, effective and well-tolerated procedure of treatment. ${ }^{22}$ According to the guidelines of the Society of American Gastrointestinal and Endoscopic Surgeons, LA is not recommended for tumors suspected to be ACC with size $>6 \mathrm{~cm} \cdot{ }^{23}$ However, some experts consider the maximum diameter could be upregulated to $8 \mathrm{~cm}$ or even $10 \mathrm{~cm}$ on the premise of $\mathrm{R} 0$ resection of tumors.

\section{$\mathrm{R} 0$ resection is crucial}

Whatever surgical approach is chosen for ACC, complete resection of tumor is necessary and vital for the prognosis., ${ }^{3,24}$ $\mathrm{R} 0$ resection is an important factor influencing oncologic outcomes. ${ }^{25}$ The overall survival rate of ACC patients undergoing incomplete resection is obviously lower than that of those achieving complete resection, and the recurrence rate after $\mathrm{R} 0$ resection is significantly lower than that after $\mathrm{R} 1$ or $\mathrm{R} 2$ resection. ${ }^{26}$ Therefore, it is vital for patients with ACC to ensure a complete and margin-negative tumor resection in the initial operation.

Although the mechanism leading to the difference in oncologic outcomes between OA and LA is still not clear, we speculate there are several possible reasons as follows. First, when the tumor is excessively stressed and pulled, ACC is easier to rupture than other types of tumor due to the soft and fragile pathologic characteristics of ACC. Since the tactile sensation of laparoscopic operation is limited compared with open approach, there are more pulling and stressing actions close to the surface of tumors during LA, which may lead to higher incidence of rupture of tumors than OA. This may be the reason why the incidence of local recurrence after LA is higher. Second, the cells of ACC could invade through the capsule and are frequently microscopically present at the surface of tumors; so, application of laparoscopic instruments close to the surface of tumor may cause more shedding of malignant cells spreading to surrounding tissues. However, it is not found in open operations where touching the surface of tumors directly is avoided because the tumors are available to be resected covered by the surrounding adipose tissue. Third, the optimal exploration of surgery field of laparoscopic operation is worse than that of open operation, and resection of surrounding tissues during LA may be incomplete compared to that in open approach. Unfortunately, some minute surrounding tissue invasion of ACC is invisible to the naked eye, and it may be why outcomes of LA for ACC with T3 are worse than that of OA. Finally, the airflow caused by application of laparoscopic pneumoperitoneum machine during laparoscopic operations may lead to diffusion of shedding tumor cells around the surgical field, increasing the possibility of recurrence.

However, some researchers insisted that laparoscopic surgery was still feasible for R0 resection of malignant or potentially malignant adrenal tumors. In a research published by Pędziwiatr et al including 52 patients receiving laparoscopic adrenalectomies for malignant or potentially malignant adrenal tumors, it was reported that $\mathrm{R} 0$ resection was achieved in $78.8 \%$ of patients (41/52), and the authors considered that laparoscopic surgery could be comparable to open approach for malignant adrenal tumors with the possibility of noninferiority. ${ }^{27}$ In our opinion, although it is still controversial whether OA is superior to LA in the rate of $\mathrm{R} 0$ resection, we believe that guarantee of $\mathrm{R} 0$ resection is crucial for the prognosis of ACC patients. 


\section{Limitations and insufficiency}

As the ACC is a rare malignant tumor with a low incidence, the number of cases included in our study is limited. All the researches which have been published so far, including this study, are retrospective comparative studies, while prospective comparative series are still lacking. The skills and experience of surgery for ACC vary among different surgeons, so the results may not be the best advice on management for every patient with ACC.

\section{Conclusion}

In summary, OA for ACC is superior to laparoscopic approach in terms of short-term oncologic prognostic factors such as DFS and rate of 2-year DFS, although OA means larger maximum diameter of tumors and less benefit during perioperation. In addition, after LA, patients are more likely to show local recurrent lesions at the first time of relapse. Taking the results of our study and the existing data of current published researches into account, we recommend OA as the gold standard of surgical treatment for ACC. Laparoscopic approach may be appropriate for selected cases of ACC without invasion of surrounding tissues, on the premise of $\mathrm{R} 0$ resection.

\section{Availability of data}

All the data were collected from the medical records of patients in PUMCH. Editors and reviewers could send e-mail to hzlipumch@163.com to obtain any data associated with the study.

\section{Author contributions}

Study concepts: HZL. Study design: GYZ. Data acquisition: GYZ, JHD. Quality control of data and algorithms: XBZ, XCW. Data analysis and interpretation: GYZ, HZL, JHD. Statistical analysis: XCW, XBZ. Manuscript drafting: GYZ, JHD. Manuscript review: HZL, XBZ, XCW. All authors contributed toward data analysis, drafting and revising the paper and agree to be accountable for all aspects of the work.

\section{Disclosure}

The authors report no conflicts of interest in this work.

\section{References}

1. Else T, Kim AC, Sabolch A, et al. Adrenocortical carcinoma. Endocr Rev. 2014;35(2):282-326.

2. Kerkhofs TM, Verhoeven RH, Van der Zwan JM, et al. Adrenocortical carcinoma: a population-based study on incidence and survival in the Netherlands since 1993. Eur J Cancer. 2013;49(11):2579-2586.
3. Ayala-Ramirez M, Jasim S, Feng L, et al. Adrenocortical carcinoma: clinical outcomes and prognosis of 330 patients at a tertiary care center. Eur J Endocrinol. 2013;169(6):891-899.

4. Fassnacht M, Johanssen S, Fenske W, et al. Improved survival in patients with stage II adrenocortical carcinoma followed up prospectively by specialized centers. J Clin Endocrinol Metab. 2010;95(11): 4925-4932.

5. Tran TB, Liou D, Menon VG, Nissen NN. Surgical management of advanced adrenocortical carcinoma: a 21-year population-based analysis. Am Surg. 2013;79(10):1115-1118.

6. Crucitti F, Bellantone R, Ferrante A, Boscherini M, Crucitti P. The Italian Registry for Adrenal Cortical Carcinoma: analysis of a multiinstituitional series of 129 patients. The ACC Italian Registry Study Group. Surgery. 1996;119(2):161-170.

7. Leboulleux S, Deandreis D, Al Ghuzlan A, et al. Adrenocortical carcinoma: is the surgical approach a risk factor of peritoneal carcinomatosis? Eur J Endocrinol. 2010;162(6):1147-1153.

8. Miller BS, Gauger PG, Hammer GD, Doherty GM. Resection of adrenocortical carcinoma is less complete and local recurrence occurs sooner and more often after laparoscopic adrenalectomy than after open adrenalectomy. Surgery. 2012;152(6):1150-1157.

9. Brix D, Allolio B, Fenske W, et al. Laparoscopic versus open adrenalectomy for adrenocortical carcinoma: surgical and oncologic outcome in 152 patients. Eur Urol. 2010;58(4):609-615.

10. Ball MW, Hemal AK, Allaf ME. International Consultation on Urological Diseases and European association of urology international consultation on minimally invasive surgery in urology: laparoscopic and robotic adrenalectomy. BJU Int. 2017;119(1):13-21.

11. Cooper AB, Habra MA, Grubbs EG, et al. Does laparoscopic adrenalectomy jeopardize oncologic outcomes for patients with adrenocortical carcinoma? Surg Endosc. 2013;27(11):4026-4032.

12. Mir MC, Klink JC, Guillotreau J, et al. Comparative outcomes of laparoscopic and open adrenalectomy for adrenocortical carcinoma: single, high-volume center experience. Ann Surg Oncol. 2013;20(5) 1456-1461.

13. Miller BS, Ammori JB, Gauger PG, Broome JT, Hammer GD, Doherty GM. Laparoscopic resection is inappropriate in patients with known or suspected adrenocortical carcinoma. World J Surg. 2010;34(6): 1380-1385.

14. Sgourakis G, Lanitis S, Kouloura A, et al. Laparoscopic versus open adrenalectomy for stage I/II adrenocortical carcinoma: meta-analysis of outcomes. J Invest Surg. 2015;28(3):145-152.

15. Donatini G, Caiazzo R, Do Cao C, et al. Long-term survival after adrenalectomy for stage I/II adrenocortical carcinoma (ACC): a retrospective comparative cohort study of laparoscopic versus open approach. Ann Surg Oncol. 2014;21(1):284-291.

16. Lombardi CP, Raffaelli M, De Crea C, et al. Open versus endoscopic adrenalectomy in the treatment of localized (stage I/II) adrenocortical carcinoma: results of a multiinstitutional Italian survey. Surgery. 2012; 152(6):1158-1164.

17. Porpiglia F, Fiori C, Daffara F, et al. Retrospective evaluation of the outcome of open versus laparoscopic adrenalectomy for stage I and II adrenocortical cancer. Eur Urol. 2010;57(5):873-878.

18. Major P, Matłok M, Pędziwiatr M, Budzyński A. Do we really need routine drainage after laparoscopic adrenalectomy and splenectomy? Wideochir Inne Tech Maloinwazyjne. 2012;7(1):33-39.

19. Conzo G, Tartaglia E, Gambardella C, et al. Minimally invasive approach for adrenal lesions: systematic review of laparoscopic versus retroperitoneoscopic adrenalectomy and assessment of risk factors for complications. Int J Surg. 2016;28(Suppl 1):S118-S123.

20. Natkaniec M, Pędziwiatr M, Wierdak M, et al. Laparoscopic transperitoneal lateral adrenalectomy for large adrenal tumors. Urol Int. 2016; 97(2):165-172.

21. Pędziwiatr M, Wierdak M, Ostachowski M, et al. Single center outcomes of laparoscopic transperitoneal lateral adrenalectomy-lessons learned after 500 cases: a retrospective cohort study. Int J Surg. 2015;20:88-94. 
22. Conzo G, Pasquali D, Gambardella C, et al. Long-term outcomes of laparoscopic adrenalectomy for Cushing disease. Int J Surg. 2014; 12(Suppl 1):S107-S111.

23. Stefanidis D, Goldfarb M, Kercher KW; Society of Gastrointestinal and Endoscopic Surgeons. SAGES guidelines for minimally invasive treatment of adrenal pathology. Surg Endosc. 2013;27(11): 3960-3980.

24. Bilimoria KY, Shen WT, Elaraj D, et al. Adrenocortical carcinoma in the United States: treatment utilization and prognostic factors. Cancer. 2008; 113(11):3130-3136.
25. Grubbs EG, Callender GG, Xing Y, et al. Recurrence of adrenal cortical carcinoma following resection: surgery alone can achieve results equal to surgery plus mitotane. Ann Surg Oncol. 2010;17(1):263-270.

26. Icard P, Chapuis Y, Andreassian B, Bernard A, Proye C. Adrenocortical carcinoma in surgically treated patients: a retrospective study on 156 cases by the French Association of Endocrine Surgery. Surgery. 1992;112(6):972-979; discussion 979-980.

27. Pędziwiatr M, Wierdak M, Natkaniec M, et al. Laparoscopic transperitoneal lateral adrenalectomy for malignant and potentially malignant adrenal tumours. BMC Surg. 2015;15:101.

\section{Publish your work in this journal}

OncoTargets and Therapy is an international, peer-reviewed, open access journal focusing on the pathological basis of all cancers, potential targets for therapy and treatment protocols employed to improve the management of cancer patients. The journal also focuses on the impact of management programs and new therapeutic agents and protocols on

\section{Dovepress}

patient perspectives such as quality of life, adherence and satisfaction. The manuscript management system is completely online and includes a very quick and fair peer-review system, which is all easy to use. Visit http://www.dovepress.com/testimonials.php to read real quotes from published authors. 